\title{
Large Leaking Descemetocele Presenting As a Traumatic Corneal Laceration
}

\author{
Logeswary K, Jemaima Che-Hamzah, Aida Zairani MZ ( $₫)$ \\ Department of Department of Ophthalmology, Faculty of Medicine, Universiti Kebangsaan Malaysia \\ Medical Centre, Jalan Yaacob Latif, Bandar Tun Razak, 56000 Cheras, Kuala Lumpur, Malaysia.
}

\begin{abstract}
A 47-year-old healthy Indonesian gentleman, presented with blurring of vision on the left eye associated with pain and redness after history of foreign body entering the eye. A general practitioner prescribed him some eye drops; however, his symptoms worsened. A corneal laceration wound measuring $0.5 \times 3.5 \mathrm{~mm}$ with a large stromal abscess measuring $9.0 \times 5.0 \mathrm{~mm}$ with flat anterior chamber was seen on the left eye. Emergency corneal T\&S revealed a large descemetocele and cyanoacrylate corneal glue was applied on the area. C\&S showed Pseudomonas aeruginosa. Patient was subsequently undergone penetrating keratoplasty as a definitive measure. This case highlights the rapid progression of pseudomonas keratitis, presented with a large descemetocele, which can be mistaken as an infected corneal laceration wound. Corneal cyanoacrylate glueing together with corneal suturing is a viable temporary method for large descemetoceles awaiting definitive surgery.
\end{abstract}

Keywords: Cyanoacrylate, descemet membrane, gram negative bacteria, keratitis, pseudomonas aeruginosa

\section{Correspondence:}

Aida Zairani Mohd. Zahidin. Department of Ophthalmology, Faculty of Medicine, Universiti Kebangsaan Malaysia Medical Centre, Jalan Yaacob Latif, Bandar Tun Razak, 56000 Cheras, Kuala Lumpur, Malaysia. Tel: +603-91455981 Fax: 03-91456733 E-mail: aidazahidin@gmail.com

\section{Introduction}

Pseudomonas aeruginosa is an opportunistic pathogen that can cause infection at various sites of the human body. Cornea is one of the ocular structure which is often involved (1). Pseudomonas aeruginosa keratitis commonly presents as a ring abscess in the cornea (2). It is normally triggered by contact lens wear or corneal trauma, and can progress rapidly leading to corneal melting with profound impairment of vision $(2,3)$.

\section{Case Report}

A 47-year-old healthy Indonesian gentleman who is a non-contact lens wearer, presented with blurring of vision on the left eye associated with pain and redness. Three days prior to presentation, he had history of foreign body entering the affected eye after drilling into a ceiling. He did not wear any protective eye wear. The eye was immediately irrigated with tap water, but he started to rub his eye due to itchiness.
Subsequently two days later, he noticed a sudden reduction in vision. A general practitioner prescribed him some eye drops, however, his symptoms worsened and he was subsequently referred to our centre.

At presentation, visual acuity in the right eye was $6 / 6$ and hand movement in the left eye. Anterior segment and fundus examination of right eye was normal. Examination of left eye revealed a suspicious corneal laceration wound measuring $0.5 \times 3.5 \mathrm{~mm}$ with a large stromal abscess measuring $9.0 \times 5.0 \mathrm{~mm}$ as shown in Figure 1. Anterior chamber was flat. CT-scan orbit did not indicate any intraocular foreign body.

Emergency corneal toilet and suturing (T\&S) with anterior chamber reformation was performed. Intraoperatively, a large perforated descemetocele was found, instead of a corneal laceration, as suspected earlier on slit lamp examination. Due to lack of cornea tissue for grafting, corneal suturing and cyanoacrylate glue was applied onto the area to regain globe integrity 


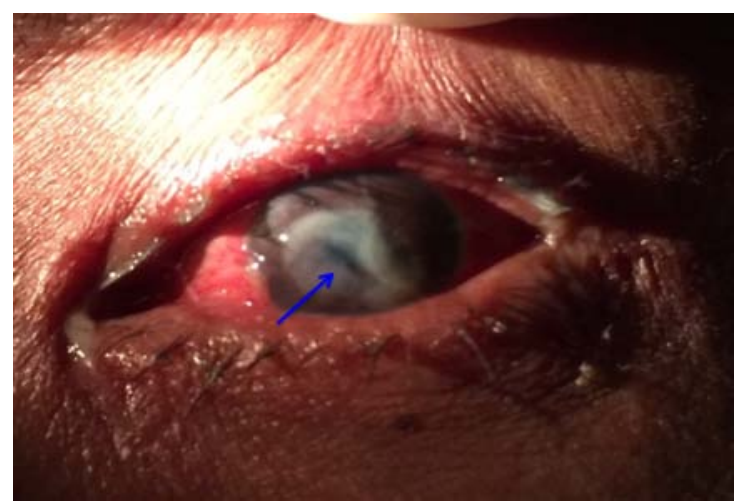

Figure 1: On presentation, anterior segment photo of the left eye showing a suspicious corneal laceration wound with large stromal abscess.

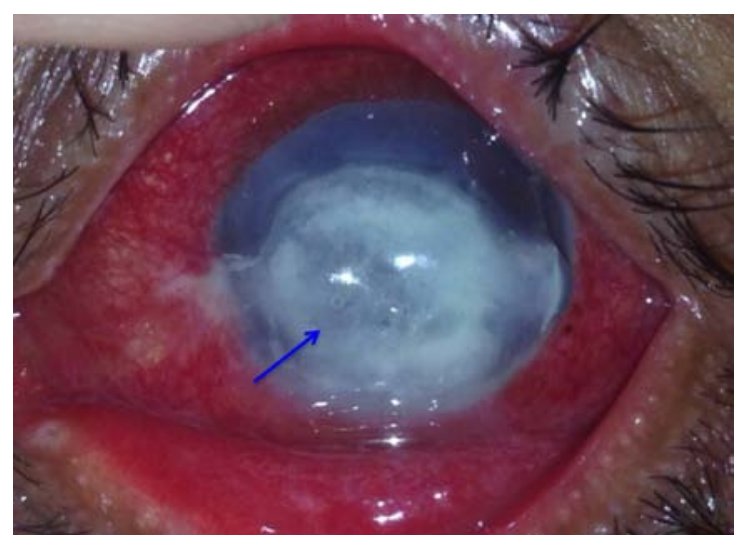

Figure 2: Day 2 postoperative image showing cyanoacrylate glue and bandage contact lens in situ.

temporarily after the debridement of surrounding stromal abscess as shown in Figure 2. Operative procedure ended with the application of bandage contact lens over the left eye.

Corneal scrapping culture and sensitivity (C\&S) revealed pseudomonas aeruginosa infection. Postoperatively, intensive topical gentamycin 0.9\% and ceftazidime 5\% were prescribed. Even though, there was no evidence of endophthalmitis, intravenous ciprofloxacin $400 \mathrm{mg}$ twice daily was started at presentation and converted to oral ciprofloxacin 750 mg twice daily for one week duration. Daily dose of oral doxycycline $100 \mathrm{mg}$ and vitamin C $500 \mathrm{mg}$ was also given to promote corneal stromal wound healing.

Due to financial constraints, patient was subsequently referred to another government tertiary eye centre for further management of his condition. There, he underwent tectonic penetrating keratoplasty as a definitive treatment measure for the large cornea perforation. His best-corrected visual acuity was 6/18 at nine months post corneal transplant, and he was able to resume his work as a contractor.

\section{Discussion}

Bacterial keratitis is a serious cause of ocular morbidity, caused by disruption of corneal epithelium continuity and integrity. Even though contact lens wear is the most common risk factor for bacterial keratitis, trauma (including chemical, thermal and foreign bodies) and contaminated ocular solution can also be contributing factors. Keratitis caused by Gramnegative bacteria is more fulminant and results in poor visual prognosis, especially in pseudomonas related keratitis (2-4).

The usual presentation is sudden onset and progress of eye pain, redness, increased tearing, blurred vision and photophobia. Examination normally reveals corneal epithelial defect and stromal infiltrate, which often assumes a "ring" configuration. Stromal necrosis, progressive thinning and/or perforation are the other reported clinical features. There is often a characteristic, "ground glass" appearance in the uninvolved areas of the cornea adjacent to the active lesion, as well as an inflammatory endothelial plaque and/or hypopyon (4).

Pseudomonas aeruginosa has many virulence factors contributing to keratitis. Beside extracellular products like protease, elastase, exoenzyme S, exotoxin A and endotoxin, this microorganism has anatomical structures like pili and flagella to facilitate invasion and colonization. Protease enzymes play an important role in corneal stroma destruction with subsequent descemetocele formation and/or corneal perforation (5). Descemetocele, the forward herniation of deeper corneal layer of descemet membrane, is a well-known complication of pseudomonas related keratitis.

Diagnosis of descemetocele is usually made if there is a clear zone within areas of thinning with descement folds at the base of the keratitis. In perforated descemetocele, the anterior chamber is flat or shallow with positive Seidel test and area of perforation is clearly seen. In some cases, the site of perforation may be small or obscured by necrotic, infiltrated, or oedematous tissue and the anterior chamber may be only minimally shallow. In our patient, he had history of trauma and presented with haziness of the cornea and flat anterior chamber. The leaking desmatocele was obscured by the necrotic and oedematous tissue, which led us to the diagnosis of infected traumatic corneal laceration. However, intraoperatively, there was no seepage of viscoelastic material through the suspected corneal laceration wound when viscoelastic was injected into anterior chamber to reform it, making the diagnosis of a leaking desmatocele more likely. In cases of infected corneal laceration, there 
will be a definite cornea defect where viscoelastic material will ooze through the defect during anterior chamber reformation.

Cyanoacrylate glueing is a useful technique as a temporary measure for treatment of infectious corneal perforations to allow delivery of antimicrobial treatment prior to a more definitive management with penetrating keratoplasty. It has the advantages of being bacteriostatic, thus inhibits polymorphonuclear lymphocytes and the production of collagenases, which may also halt the corneal melting process. Definitive surgery can be planned under a more controlled manner once inflammation of the eye has been reduced and the integrity of the globe restored (6). This is especially useful in cases where donor cornea tissue is not readily available, such as in this patient. In cases of large perforations and associated surrounding tissue necrosis, suturing the perforation with 10/0 Nylon suture is a useful method to provide better structural support to the perforation prior to stabilization with cornea glueing.

Tectonic penetrating keratoplasty is one of the suitable definitive surgical treatments available to replace the destructive corneal tissue that has been lost due to disease process especially in the cases of large cornea tissue lost like in our case. It is advisable for large corneal perforation measuring more than $3 \mathrm{~mm}$. However, it is recommended that temporary measures such as corneal glue and bandage contact lens should be considered first until the active phase of infection is controlled prior to performing the definitive penetrating keratoplasty (7). This approach is very effective in restoring the anterior chamber integrity and eventually saving the globe for better vision (8).

\section{Conclusion}

This case highlights the rapid progression of pseudomonas keratitis, which presented with a large descemetocele in a patient, post trauma. Cornea glueing with cyanoacrylate glue together with corneal suturing is a viable temporary method even for a large descemetocele awaiting definitive surgery.

\section{References}

1. Tang A, Caballero AR, Marquart ME, O'Callaghan RJ. Pseudomonas aeruginosa Small Protease (PASP), a Keratitis Virulence Factor PASP, a Pseudomonas Virulence Factor. Invest Ophthalmol Vis Sci 2013; 54(4): 2821-8.

2. Oka N, Suzuki T, Ishikawa E, et al. Relationship of Virulence Factors and Clinical Features in Keratitis Caused by Pseudomonas aeruginosa Investigative ophthalmology \& visual science. Invest Ophthalmol Vis Sci 2015; 56(11): 6892-8.

3. Ishikawa E, Suzuki T, Yamaguchi S, Inoue $\mathrm{T}$, Uno $\mathrm{T}$, Ohashi Y. Serrated margins in Pseudomonas aeruginosa keratitis. Case Rep Ophthalmol 2013; 4(2): 12-5.

4. Weed MC, Rogers GM, Kitzmann AS, Goins KM, Wagoner MD. Vision Loss after Contact Lens-Related Pseudomonas Keratitis. Website: http://www.EyeRounds.org/cases/171-

pseudomonas-keratitis.htm. Last accessed on 24/06/2013.

5. Twining SS, Davis SD, Hyndiuk RA. Relationship between proteases and descemetocele formation in experimental Pseudomonas keratitis. Curr Eye Res 1986; 5(7): 503-10.

6. Vote BJ, Elder MJ. Cyanoacrylate glue for corneal perforations: a description of a surgical technique and a review of the literature. Clin Exp Ophthalmol 2000; 28(6): 437-42.

7. Cheung N, Sayegh RR, Gupta PC. Management of Descemetocele and Corneal Perforation. Website:-eyewiki.aao.org. Last accessed on 05/01/2017.

8. Jonas JB, Rank RM, Budde WM. Tectonic sclerokeratoplasty and tectonic penetrating keratoplasty as treatment for perforated or predescemetal corneal ulcers. Am J Ophthalmol 2001; 132(1): 14-8. 